\title{
A Texture Based Shoe Retrieval System for Shoe Marks of Real Crime Scenes
}

\author{
Francesca Dardi, Federico Cervelli`, and Sergio Carrato \\ Dept. Electrical, Electronic and Information Engineering (DEEI) \\ University of Trieste, 34100 Trieste, Italy \\ federico.cervelli@deei.units.it
}

\begin{abstract}
Shoeprints found on the crime scene contain useful information for the investigator: being able to identify the make and model of the shoe that left the mark on the crime scene is important for the culprit identification. Semi-automatic and automatic systems have already been proposed in the literature to face the problem, however all previous works have dealt with synthetic cases, i.e. shoe marks which have not been taken from a real crime scene but are artificially generated with different noise adding techniques.

Here we propose a descriptor based on the Mahalanobis distance for the retrieval of shoeprint images. The performance test of the proposed descriptor is performed on real crime scenes shoe marks and the results are promising.
\end{abstract}

\section{Introduction}

Crime scene investigation is the starting point towards the identification of the culprit: only a great care and a careful analysis of the scene of crime allow the investigators to gain precious information about the criminal act. Among the activities to be performed by the crime scene experts we can recall the detailed documentation of the scene status, the search for fingerprints, shoeprints, biological fluids, chemicals and fire arms ammunition, and the collection of the items pertaining the crime for a later and deeper analysis in the laboratory [1].

In particular, shoe marks have a key role to understand the crime [2] and contain valuable information that can help investigators to unveil both the dynamics and the actors of the criminal action [3]: when no suspects or few elements are available to track investigation, knowing the make and the brand of the shoe sole that left the shoe mark on the scene can point a path; on the other side, if a suspect is given, the shoe mark can be compared to the shoes owned by the suspect, in order to formulate a statement about his or her involvement in the crime 4.

In this paper we deal with the first case: one or more shoe marks have been found on the crime scene and the make and model of the originating shoes must be found.

\footnotetext{
^ Corresponding author.
} 
The paper is organized as follows: in Sec. 2 we give the current state of the art of the research on automatic shoeprint matching, in Sec. 3 we describe the proposed descriptor and metrics, and in Sec. 4 we discuss the results. Finally in Sec. 5 we draw the conclusions and point out the future work.

\section{Shoeprint Image Matching}

We are interested in finding the make and the model of the shoe which produced a given shoe mark on the crime scene. In order to satisfy this request two different approaches can be followed:

1. a forensic shoeprint examiner analyzes the shoe mark and looks for the corresponding shoe on both digital and paper catalogs;

2. the crime scene shoe mark is queried using a reference database (DB) of known shoes, thanks to a content based image retrieval system, and the results of the query are then analyzed by the expert.

Some semi-automatic systems have been proposed to face the problem. In 567 . shoe soles (and shoeprints more in general) are classified by a human expert which describes them with a series of geometric patterns.

Some automatic systems have also been proposed, but the field is still young. In 8] soles and shoeprints are described by a series of patterns automatically generated through a series of erosion-dilation steps. The Fourier components of the patterns are then calculated and chosen in order to be processed by a final classification through a neural network. Real crime scene shoeprints are used, but no performance results are given.

Fractals and mean square error are employed in [9] to represent and compare shoeprints, respectively. The DB is composed of 145 full print images, and is queried by the same images composing the DB but with added noise, rotation and translation. A 13 pixel translation result in a $10 \%$ accuracy of the results, and the algorithm needs the same size of the images for the mark and DB.

Fourier transform is implemented in 10. The DB contains 476 full prints, which are correctly matched in the first $5 \%$ of the sorted DB patterns with an $85 \%$ score. Noisy images are not considered, and images are matched on themselves, to prove rotational and translational invariance.

Phase only correlation is employed in [11] to compare partial shoeprints produced starting from a 100 elements DB: these items are corrupted with noise or are added with some background texture images to simulate real case scenarios. The obtained simulated shoe marks are then queried to the DB, showing a satisfying performance.

More recently in 12 Hu's moment invariants are used on a DB containing 500 images, which are synthetically corrupted by noise and then queried against the full DB. Rotational robustness is tested in the range from $-90^{\circ}$ to $+90^{\circ}$. The first returned image correctly matches the query image $99.4 \%$ of the time.

A Maximally Stable Extremal Region (MSER) detector is used in [13] to identify the features of the shoeprint and the Scale Invariant Feature Transform 
(SIFT) algorithm is employed to describe them. After this first step a shorter list remains, and a finer search proceeds by comparing the shoeprints through a modified constrained spectral correspondence method. A query on the 374 elements in the DB gives a $92 \%$ top-eight rank. Also in this case the queried shoeprints are different print of varying quality of the same shoe model, i.e. they are not shoe marks coming from real crime scenes.

In this paper we have tested a new method over a reference DB of 87 known sole prints. Differently from all previous works, the query images used to evaluate the performance of the system are marks from real crime scenes.

\section{The Proposed Shoeprint Image Retrieval System}

A shoe sole is made with a distinguishing pattern which, in principle, can be thought as composed by a texture and a series of unique shapes (e.g. logos and other structures). Here, we focus on a proposal for the textured region descriptor and its recognition system. Obviously a full system would combine these results with those of a second algorithm based on the analysis of the unique shapes quoted above, in order to proceed with a finer search on the reduced cardinality DB.

\subsection{Descriptor Selection and Calculation}

In the following we describe a novel descriptor used to represent the texture region appearance in shoe sole marks found at crime scenes, on different types of background. This representation is based on the geometrical structures of objects, as proposed in [14] to detect human figures. As we can not assume particular pixel value patterns for objects like shoe marks found at crime scenes, we focus on geometrical structures observed as distances between pixel value patterns. Here, the pixel values are just labels making some regions stand out against other regions, because the variety of colors and textures of possible background where shoe marks can be found is enormous. Conversely, the relative positions and shapes of different parts in the sole print texture region are common for a given shoe mode 1 . We select the proposed descriptor in order to focus more on geometrical entities rather than on pixel values themselves. So we extract the geometrical structures of our target object: this method is similar to edge based object recognition methods, but methods based only on edge detection are generally too sensitive to local information and are not robust against noise. In this work, we actually apply the proposed descriptor on gray scale shoeprint images either directly or after performing edge detection, in order to both overcome the aforementioned disadvantages of edge detection methods and improve recognition rate.

We briefly review the descriptor calculation. We divide a gray scale image area that we are interested in, into several sub-regions or blocks, and calculate

${ }^{1}$ We are not taking into account counterfeits reproducing the sole pattern of famous shoe makers. 
the distances between all possible block pairs. More in detail, for an image $x(s, t)$ of size $m \times n$, we divide the area into small blocks of size $p \times q$. As a result we have $M$ blocks in the horizontal direction and $N$ blocks in the vertical direction. We first identify each block with a number ranging from 1 to $M N$ in order to distinguish them as block $X_{1}, X_{2}, \ldots, X_{M N}$, and we compute the average vector, $\bar{x}_{l}$, and variance $\sigma_{l}^{2}$ for each block $X_{l}$.

We define the Mahalanobis distance 15] $d(i, j)$, with $i, j=1, \ldots, M N$, between all possible block pairs:

$$
d(i, j)=\left(\bar{x}_{i}-\bar{x}_{j}\right)\left(\sigma_{i}^{2}+\sigma_{j}^{2}\right)^{-1}\left(\bar{x}_{i}-\bar{x}_{j}\right),
$$

in order to determine the Mahalanobis map $\mathcal{M}$ :

$$
\mathcal{M}=\left(\begin{array}{cccc}
0 & d(1,2) & \cdots & d(1, M N) \\
d(2,1) & 0 & \cdots & d(2, M N) \\
\vdots & \vdots & \ddots & \vdots \\
d(M N, 1) & d(M N, 2) & \cdots & 0
\end{array}\right)
$$

We used a $4 \times 4$ pixel block size for the calculation, for a total amount of $25 \times$ 25 blocks (as our images are $100 \times 100$ pixel size wide).

Finally the descriptor of the mark and of the shoe sole in the DB is calculated as the Power Spectral Density (PSD) of $\mathcal{M}$, obtained as in 10. In order to take care of the different noise sources affecting the shoe marks, the PSD is computed on the Mahalanobis map either calculated directly on the gray scale values of the image, or after the application of a suitable edge detection algorithm.

\subsection{Similarity Metrics}

In order to compare the input image (i.e. the shoe mark) with each of the images of the DB, a measure of similarity between the evaluated descriptors of the two images is required. The descriptor is calculated for each sole print image in the reference DB (corresponding to a different shoe make and model), and for the input shoe mark image; then the similarity measure between the descriptor of the mark and the descriptor of each item in the DB is computed and used to rank the results. The highest rank results are then supposed to be shown to a human expert who will select the database shoe that matches the shoe mark image (if a match exists).

The proposed measure of similarity between the shoe mark image and the image in the $\mathrm{DB}$ is their correlation coefficient. For $2 \mathrm{D}$ signals of size $r \times s$, $f_{i}(x, y)$, the correlation coefficient, $r_{i, j}$, is calculated using [17]:

$$
\begin{gathered}
\hat{f}_{i}(x, y)=\left[f_{i}(x, y)-\text { mean }\left(f_{i}\right)\right] / \operatorname{std}\left(f_{i}\right) \\
r_{i, j}=\frac{1}{r s} \sum_{y=1}^{r} \sum_{x=1}^{s} \hat{f}_{i}(x, y) \hat{f}_{j}(x, y)
\end{gathered}
$$

where mean $\left(f_{i}\right)$ is the average value of signal $f_{i}(x, y)$, and $\operatorname{std}\left(f_{i}\right)$ is its standard deviation. 


\section{Results and Discussion}

Performance testing of the proposed method was performed on a known shoe soles DB querying real marks coming from real crime scenes. The items were collected from the ENFSI Working Group on Marks website 16] and then adapted as explained in the following.

Starting from the images available on the website, we built a reference DB made up of 87 known shoe soles, and a shoe marks list to query, made up of 30 items. The images were converted to gray scale and subjected to a rough resize and rotation, just in order to have an approximate correspondence in size and orientation between the soles and the marks.

Then one or more zones of interest of $100 \times 100$ pixel size were cropped and used for the test; in Fig. 1] some of the shoe soles and marks are shown. Some of the shoes from the reference DB were cropped in more than one image, in order to increase the reference DB dimension for testing purposes, Fig. 2.
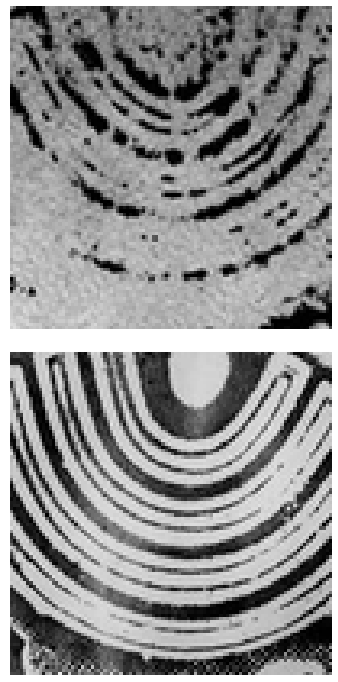
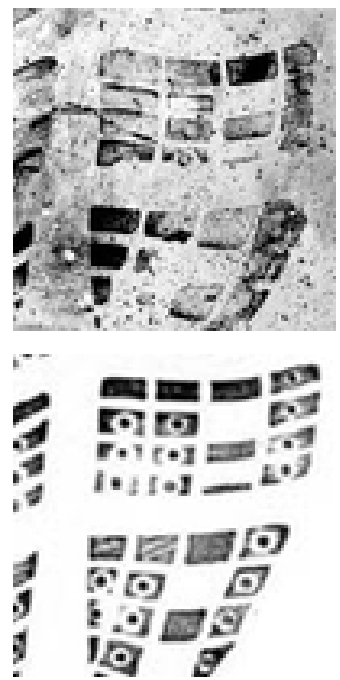
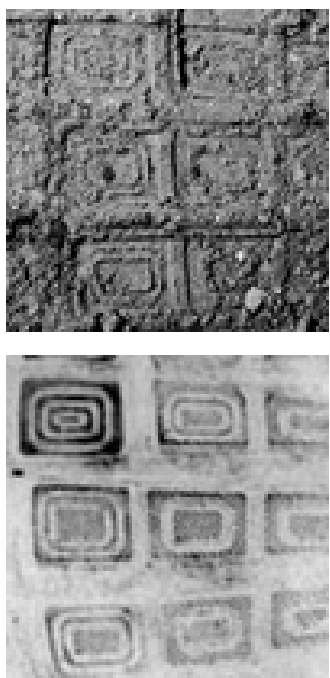

Fig. 1. Examples of some shoe marks (top), and the expected matching shoes in the reference DB (bottom)

The descriptor described in Sec. 3.1 is computed either on the gray scale values of the image or after the application of a suitable edge detection algorithm. Fig. 3 shows the ranking results for each shoe mark queried, in case of both luminance values and two different edge detection algorithms (Laplacian and Canny). As can be seen, the $73 \%$ of the real case marks were found in the top- 10 and their $100 \%$ in the top-19 shoes in at least one of the three used methods,

${ }^{2}$ European Network of Forensic Science Institutes, website: http://www.enfsi.eu/ 

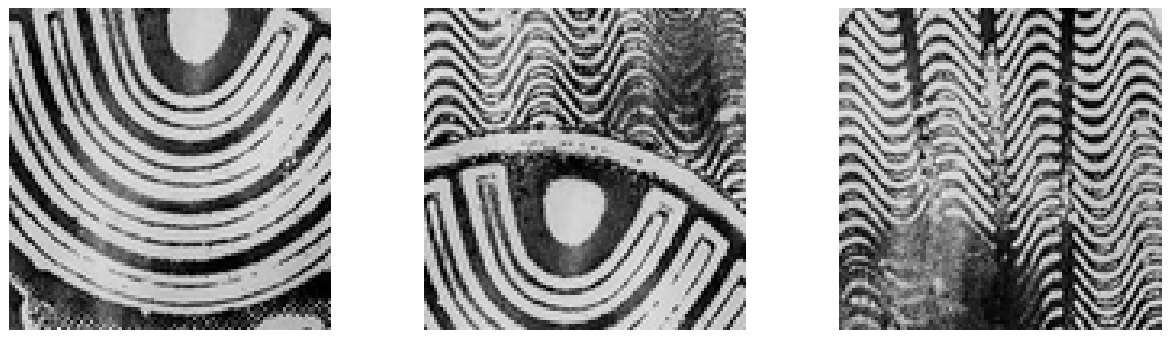

Fig. 2. Some shoeprints in the reference DB are cropped in more than one region, to increase the items number in the reference DB

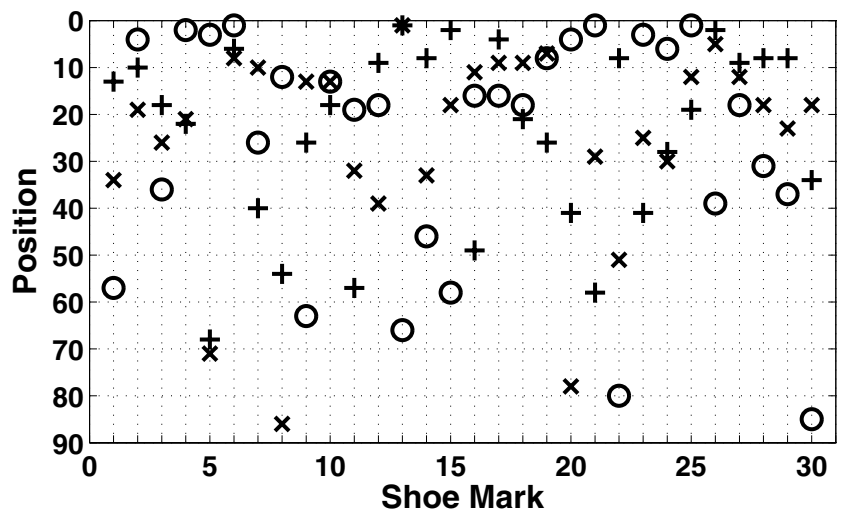

Fig. 3. The performance results are shown using the luminance image (o), or using images processed by Laplacian edge detector $(+)$, and Canny edge detector $(\times)$

thus correctly excluding from the query results $89 \%$ and $78 \%$ of the known shoes DB, respectively. Fig. 4 is the same as Fig. 3 with only the best match shown for each show mark.

Finally, Fig. 5 represents the cumulative matching characteristics (CMC) 18 of each technique taken separately, while 6 shows the CMC obtained considering only the best ranking technique for each shoe mark. In both cases the horizontal axis of the graph is the percentage of the reference DB reviewed and the vertical axis is the probability of a match.

It has to be noted that no restoration and enhancement preprocessing methods have been employed, and that, as can be seen from Fig. 1, the repositioning, resizing and rotation during the shoe DB and marks list adaptation were performed without particular care; thus the results show that the system is able to face small rotations and has the ability to detect correlations even in case of shift and only partial overlapping between regions in the shoe mark and reference DB images. 


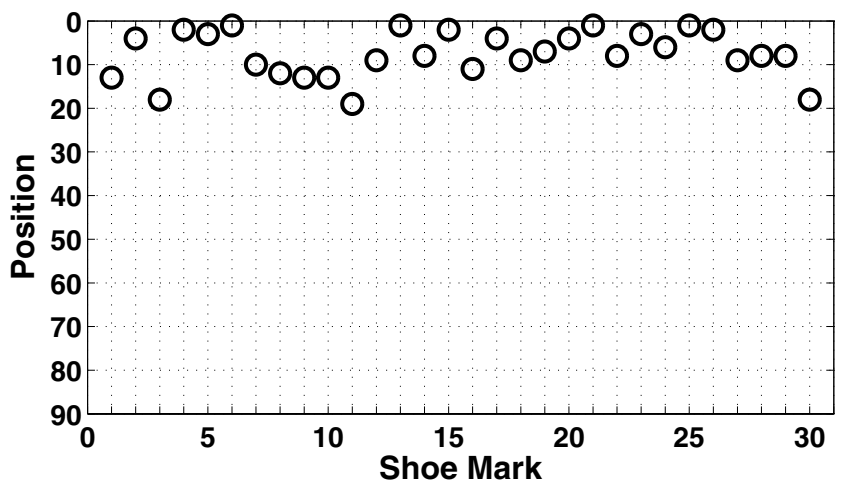

Fig. 4. Same as Fig. 3 with only the best match shown

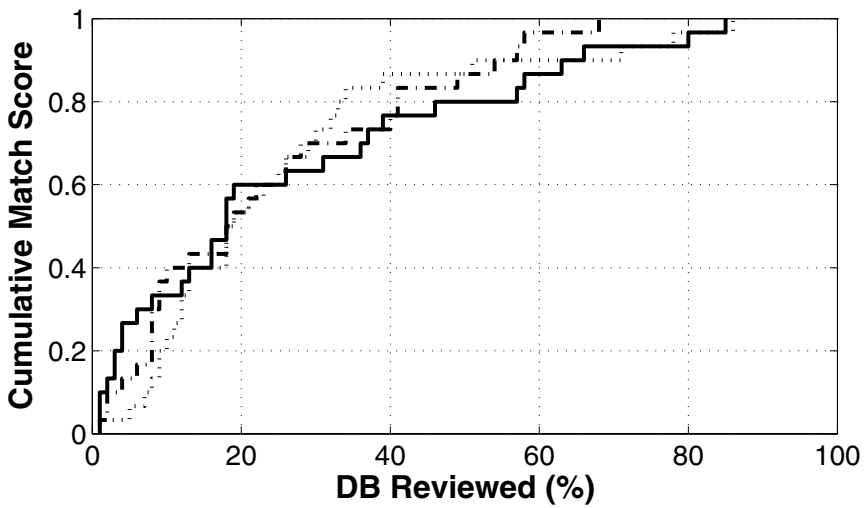

Fig. 5. Cumulative match score results using the luminance image $(-)$, or using images processed by Laplacian edge detector (- -), and Canny edge detector $(\cdots)$

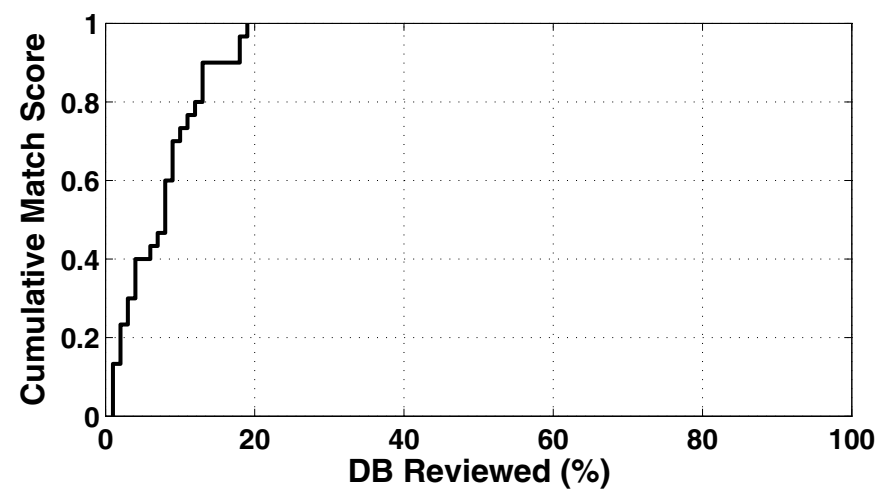

Fig. 6. Cumulative match score resulting by considering only the highest ranking technique for each shoe marks 

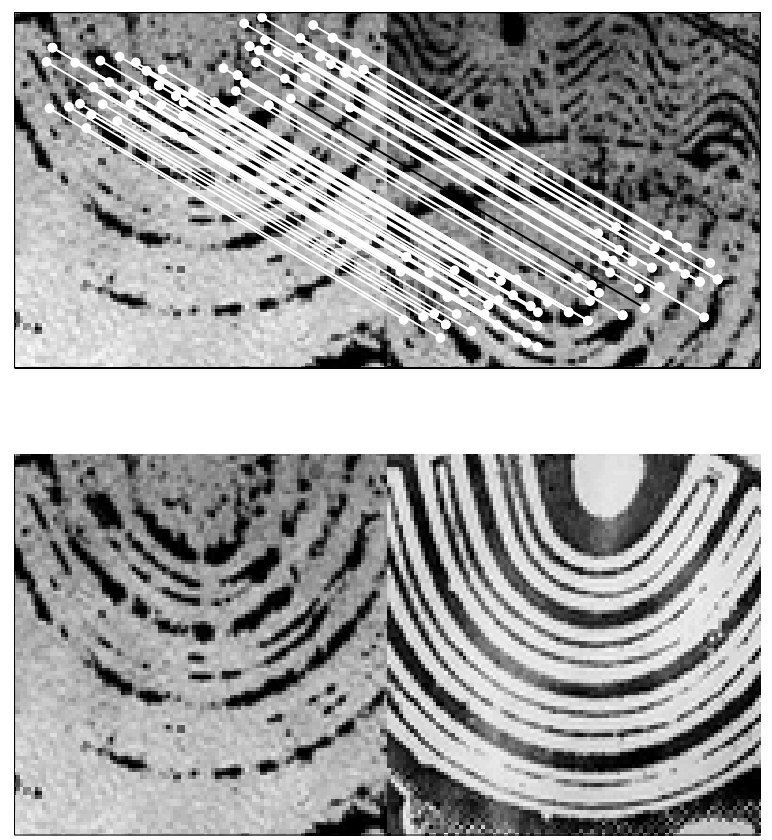

Fig. 7. The SIFT algorithm correctly matches (white lines show the corresponding features) the same shoe mark region with itself after a small translation (top), but is not able to match a shoe mark on the reference DB (bottom)

We would like to stress that this approach is taken to test the algorithm, while an operator oriented test would be instead performed using a different point of view, i.e. the one where the interest is in obtaining the right shoe, instead of the right region of the shoe.

Up to now none has reported on real shoe marks DB studies, thus none else has employed our known shoes DB and shoe marks list. The comparison of real crime scene shoe marks is a really demanding task, which can be simulated with great difficulty. We preliminary tested a SIFT matching algorithm [19], similar to the one employed in [13], on our marks and reference DB, and found that this algorithm is not likely to succeed without preprocessing in this case, Fig. 7.

The absence of a de facto standard reference DB with real crime scene marks seems to be, presently at least, a serious limit to any kind of performance comparison test. Thus, we have compared our system and the ones detailed in [10] and 11] on both synthetic and real shoe marks, showing that simulated shoe marks are not suited to test a footwear retrieval system aimed at finding the shoe make and model of a shoe mark found on the crime scene 20]: the algorithm in [11] outperformed the others on the synthetic test set, but it was on par with them when considering the results obtained on the real shoe marks set. 


\section{Conclusions and Future Work}

In this paper, we consider a descriptor based on geometrical pixel structures, i.e. the Mahalanobis map applied following three different strategies, to recognize textures of shoe marks found at the crime scenes: such a performance evaluation has never been done in previous works, at the best of our knowledge. Results are encouraging, given the fact that no additional restoration and enhancement techniques have been used before querying the reference DB. However, we must note that a direct comparison with other works is not possible, given the lack of a standard reference DB for testing.

We believe we need to estimate the quality and nature (e.g. on sand, in blood, in the mud, etc.) of a shoe mark before it is fed into the process, to automatically select the best of the three proposed strategies required to optimize the performance of the system. Segmentation techniques could also be employed to separate textured regions from unique shapes (e.g. marks, logos, etc.). Thus future work will be devoted both to the investigation of suitable restoration and enhancement techniques for shoe mark images, and to enhance the performance of the system by combining the results of the proposed texture based algorithm with those of a suitable details extraction algorithm.

\section{References}

1. James, S.H., Nordby, J.J.: Forensic Science: an introduction to scientific and investigative techniques, 2nd edn. CRC Press, Boca Raton (2005)

2. Girod, A.: Shoeprints: coherent exploitation and management. In: European Meeting for Shoeprint Toolmark Examiners, The Netherlands (1997)

3. Bodziak, W.J.: Footwear impression evidence: detection, recovery and examination, 2nd edn. CRC Press, Boca Raton (1999)

4. ENFSI WGM Conclusion Scale Committee: Conclusion scale for shoeprint and toolmarks examiners. J. Forensic Ident. 56, 255-265 (2006)

5. Girod, A.: Computerized classification of the shoeprints of burglars' shoes. Forensic Sci. Int. 82, 59-65 (1996)

6. Sawyer, N.: SHOE-FIT a computerized shoe print database. In: Proc. Eur. Convention Secur. Detect., pp. 86-89 (1995)

7. Ashley, W.: What shoe was that? The use of computerized image database to assist in identification. Forensic Sci. Int. 82, 7-20 (1996)

8. Geradts, Z., Keijzer, J.: The image-database REBEZO for shoeprint with developments for automatic classification of shoe outsole designs. Forensic Sci. Int. 82, 21-31 (1996)

9. Bouridane, A., Alexander, A., Nibouche, M., Crookes, D.: Application of fractals to the detection and classification of shoeprints. In: Proc. Int. Conf. Image Processing, vol. 1, pp. 474-477 (2000)

10. De Chazal, P., Flynn, J., Reilly, R.B.: Automated processing of shoeprint image based on the fourier transform for use in forensic science. IEEE Trans. Pattern Analysis Machine Intelligence 27, 341-350 (2005)

11. Gueham, M., Bouridane, A., Crookes, D.: Automatic recognition of partial shoeprints based on phase-only correlation. In: Proc. Int. Conf. Image Processing, vol. 4, pp. 441-444 (2007) 
12. Algarni, G., Amiane, M.: A novel technique for automatic shoeprint image retrieval. Forensic Sci. Int. 181, 10-14 (2008)

13. Pavlou, M., Allinson, N.M.: Automated encoding of footwear patterns for fast indexing. Image Vision Computing 27, 402-409 (2009)

14. Utsumi, A., Tetsutani, N.: Human detection using geometrical pixel value structures. In: Proc. 5th IEEE International Conference on Automatic Face and Gesture Recognition, pp. 39-44 (2002)

15. Gonzalez, R.C., Woods, R.E.: Digital image processing, 3rd edn. Pearson Prentice Hall, Upper Saddle River (2008)

16. Website: http://www.intermin.fi/intermin/hankkeet/wgm/home.nsf/

17. Russ, J.C.: The image processing handbook, 2nd edn. CRC Press, Boca Raton (2005)

18. Phillips, P.J., Moon, H., Rizvi, S.A., Rauss, P.J.: The FERET evaluation methodology for face-recognition algorithms. IEEE Trans. Patt. Anal. Mac. Intell. 22, 1090-1104 (2000)

19. Mikolajczyk, K., Tuytelaars, T., Schmid, C., Zisserman, A., Matas, J., Schaffalitzky, F., Kadir, T., Van Gool, L.: A comparison of affine region detectors. Int. J. Computer Vision 65, 43-72 (2005)

20. Cervelli, F., Dardi, F., Carrato, S.: Towards an automatic footwear retrieval system for crime scene shoe marks: Comparison of different methods on synthetic and real shoe marks. To be published in $6^{t h}$ Int. Symp. on Image and Signal Processing and Analysis (2009) 